\title{
Response to Glucagon in Small-for-dates Hypoglycaemic and Non-hypoglycaemic Newborn Infants
}

\author{
MARTHE A. LE DUNE \\ From the University Department of Child Health, and the Queen Mother's Hospital, Yorkhill, Glasgow \\ Le Dune, M. A. (1972). Archives of Disease in Childhood, 47, $754 . \quad$ Response \\ to glucagon in small-for-dates hypoglycaemic and non-hypoglycaemic \\ newborn infants. 32 glucagon tests with insulin studies have been performed in \\ hypoglycaemic and non-hypoglycaemic small-for-dates infants. The results suggest \\ that glycogen depletion is an important factor in neonatal hypoglycaemia, and varies \\ with the degree of hypoglycaemia. Hyperinsulinism was found in a proportion \\ of hypoglycaemic infants, though there did not appear to be any correlation between \\ the degree of hyperinsulinism and the severity of the glycogen depletion. Glucagon \\ is not recommended as a therapeutic tool in neonatal hypoglycaemia.
}

The aetiology of hypoglycaemia in the small-fordates infant has not been fully explained. The pathological studies of Shelley (1964) and Dawkins (1964) suggested that a reduced level of hepatic glycogen was an important part of the mechanism. This finding was supported by the work of Cornblath and Schwartz (1966a, b) and Shelley and Neligan (1966). More recently Blum et al. (1969) have questioned this finding as being related to the hypoglycaemia, and studied the response to intravenous glucagon in small-for-dates and normalfor-dates infants. They concluded from their study that, 'depletion of hepatic glycogen stores plays no significant role in the genesis of hypoglycaemia in small-for-dates infants'.

In view of these conflicting opinions a study was designed to investigate the response to glucagon in a group of hypoglycaemic and non-hypoglycaemic small-for-dates infants, and to estimate serially the plasma insulin. In this way it was hoped to show the glycogen depletion, and also to assess glucagon as a possible therapeutic tool in this situation. It must, however, be remembered that glucagon has many effects apart from the stimulation of blood glucose release from the liver by glycogenolysis. Milner and Wright (1967) showed that after the intravenous injection of glucagon, there was a progressive rise in the true blood glucose, plasma insulin, and growth hormone, and a fall in non-esterified fatty acids.

Clearly from the practical point of view there

Received 10 October 1971. would be some advantages in using glucagon therapeutically. It could be given intramuscularly by a nurse and therefore might rule out the necessity of giving $50 \%$ glucose intravenously, and the necessity for prolonged intravenous therapy with its attendant risks.

\section{Material}

A total of 32 infants was studied. All were born either in the Queen Mothers' Hospital, Stobhill General Hospital, or Robroyston Hospital, Glasgow. Infants below the 5th centile in weight on the Aberdeen Intrauterine Growth Chart of Tanner and Thomson (1970) before the correction was made for maternal stature were included in this series. A faint or zero recording on the Dextrostix test paper within 6 hours of birth had also to be obtained. Twins, and singletons with congenital abnormalities or hypothermia, were all excluded.

\section{Procedure}

When a Dextrostix test gave a faint or zero reading the usual practice in the department was to take a specimen of blood by heel-prick for estimation of true blood glucose, and to give an intravenous injection of $50 \%$ glucose in a dose of $1.0 \mathrm{~g} / \mathrm{kg}$ while laboratory confirmation of the hypoglycaemia was awaited. In this series glucagon was used as the therapeutic agent to raise blood sugar. In practice, the glucagon tests were carried out between the 4th and 6th hours of life, except in two cases where the initial Dextrostix gave a zero reading and even earlier testing was therapeutically indicated. The procedure was carefully explained to the mothers and was pursued only when the mothers freely consented. 
Blood was taken by heel-prick for a fasting true blood glucose and plasma insulin. An intramuscular injection of glucagon in a dose of $30 \mu \mathrm{g} / \mathrm{kg}$ was given. Blood was then taken by heel-prick 10,20,45, 60, and 90 minutes after the injection for further estimations of true blood glucose and plasma insulin.

The infants were kept under constant supervision, and regular checks with Dextrostix were carried out during the test period. If no definite colour change was obtained on the Dextrostix by the end of the first hour of the glucagon test, then the test was terminated. An intravenous injection of $50 \%$ glucose was given in a dose of $1.0 \mathrm{~g} / \mathrm{kg}$ into the smallest available peripheral vein. The infusion was then continued with a $10 \%$ glucose solution via a scalp vein, if the true blood glucose was $20 \mathrm{mg} / 100 \mathrm{ml}$ or below at the end of the first hour of the test. The infusion was continued for a variable period until such time as the infant was able to maintain alone a normal blood glucose level. The tests had to be terminated in this manner in 5 infants in the hypoglycaemic group. Oral feeding was not started till all tests were completed.

Glucagon tests were repeated at the end of the first week of life in 10 infants who had been hypoglycaemic, where the initial true blood glucose level had been 20 $\mathrm{mg} / 100 \mathrm{ml}$ or below.

Biochemical methods. True blood glucose was estimated by a glucose oxidase method developed in the Biochemestry Department of the Royal Hospital for Sick Children based on the method of Marks (1959). The blood was collected into fluoride and estimated immediately. The plasma immunoreactive insulin was estimated by the double antibody method of Hales and Randle (1963). The blood was collected into heparin and separated immediately. The plasma was then deep frozen, and the specimens estimated in batches. All estimations were in duplicate.

\section{Results}

Hypoglycaemia was defined as a fasting true blood glucose of $20 \mathrm{mg} / 100 \mathrm{ml}$ or below. On this basis the infants were divided into two groups.
1. Hypoglycaemic. A group of 15 infants with an initial fasting true blood glucose of $20 \mathrm{mg} / 100 \mathrm{ml}$ or below.

2. Non-hypoglycaemic. A group of 17 infants with an initial fasting true blood glucose above $20 \mathrm{mg} / 100 \mathrm{ml}$.

Table I shows that the true blood glucose levels are lower $(P<0.001)$ at all times throughout the glucagon tests in the hypoglycaemic group of infants. As an expression of glucose release from the liver after glucagon stimulation, the maximum true blood glucose level was taken whenever it occurred throughout the 90 -minute test period. In the hypoglycaemic group the mean maximum true blood glucose is lower $(\mathrm{P}<0.001)$.

Fig. 1 shows the correlation of the initial fasting true blood glucose with the maximum true blood glucose in the 15 hypoglycaemic infants after glucagon stimulation $(r=0.638 ; P<0.02)$. The correlation between the initial true blood glucose and the maximum rise true blood glucose was not significant $(r=0.433)$, and no correlation was found between the fasting and maximum insulin levels, the initial fasting true blood glucose and the maximum plasma insulin, the maximum true blood glucose and the maximum insulin, or the maximum insulin and the maximum rise true blood glucose estimations.

Table II shows the plasma insulin levels throughout the glucagon tests in the hypoglycaemic and non-hypoglycaemic group of infants. The fasting and mean maximum insulin levels are higher $(P<0.05$ and $<0.05)$ in the hypoglycaemic group. Fig. 2 shows the maximum insulin levels in each of the glucagon tests in the hypoglycaemic and non-hypoglycaemic groups. The mean maximum insulin level was higher $(P<0.05)$ in the hypoglycaemic group. These results are the same as in a previous study (Le Dune, 1971)

TABLE I

Comparison of Glucagon Tests i, Hypoglycaemic and Non-hypoglycaemic Small-for-dates Infants, True Blood Glucose $(\mathrm{mg} / 100 \mathrm{ml})$

\begin{tabular}{|c|c|c|c|c|c|c|c|c|}
\hline \multirow[b]{2}{*}{ Small-for-dates Infants } & \multirow[b]{2}{*}{ No. } & \multicolumn{6}{|c|}{ Time (min) After Injection of Glucagon $(30 \mu \mathrm{g} / \mathrm{kg})$} & \multirow[b]{2}{*}{$\begin{array}{l}\text { Maximum } \\
\text { True Blood } \\
\text { Glucose } \\
\text { Mean } \pm \text { SD } \\
\text { (range) }\end{array}$} \\
\hline & & $\begin{array}{c}\text { Fasting } \\
\text { Mean } \pm \text { SD } \\
\quad \text { (range) }\end{array}$ & $\underset{\substack{\text { Mean } \\
(\text { range })}}{S D}$ & $\underset{\substack{\text { Mean } \pm S D}}{20}$ & $\underset{\substack{\text { Mean } \\
\text { (range) }}}{45}$ & 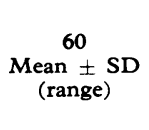 & $\underset{\substack{\text { Mean } \pm \\
\text { (range) }}}{90}$ & \\
\hline $\begin{array}{l}\text { Hypoglycaemic group } \\
\text { Non-hypoglycaemic group } \\
\text { Significance of difference }\end{array}$ & $\begin{array}{l}15 \\
17 \\
P\end{array}$ & $\begin{array}{l}10 \cdot 2 \pm 5 \cdot 3 \\
\quad(5-20) \\
31 \cdot 0 \pm 9 \cdot 6 \\
(22-57) \\
<0 \cdot 001\end{array}$ & $\begin{array}{l}17 \cdot 9 \pm 10 \cdot 8 \\
(5-38) \\
40 \cdot 2 \pm 11 \cdot 6 \\
(25-68) \\
<0 \cdot 001\end{array}$ & $\begin{array}{l}24 \cdot 3 \pm 16 \cdot 0 \\
(5-50) \\
51 \cdot 2 \pm 14 \cdot 4 \\
(30-82) \\
<0 \cdot 001\end{array}$ & $\begin{array}{l}34 \cdot 9 \pm 18 \cdot 7 \\
(5-66) \\
66 \cdot 4 \pm 17 \cdot 0 \\
(31-90) \\
<0 \cdot 001\end{array}$ & $\begin{array}{l}30 \cdot 9 \pm 18 \cdot 2 \\
(5-68) \\
70 \cdot 2 \pm 19 \cdot 4 \\
(32-95) \\
<0 \cdot 001\end{array}$ & $\begin{array}{l}29 \cdot 3 \pm 16 \cdot 1 \\
(5-54) \\
64 \cdot 9 \pm 21 \cdot 3 \\
(29-105) \\
<0 \cdot 001\end{array}$ & $\begin{array}{l}35 \cdot 3 \pm 19 \cdot 8 \\
(5-68) \\
73 \cdot 2 \pm 19 \cdot 5 \\
(32-105) \\
<0 \cdot 001\end{array}$ \\
\hline
\end{tabular}




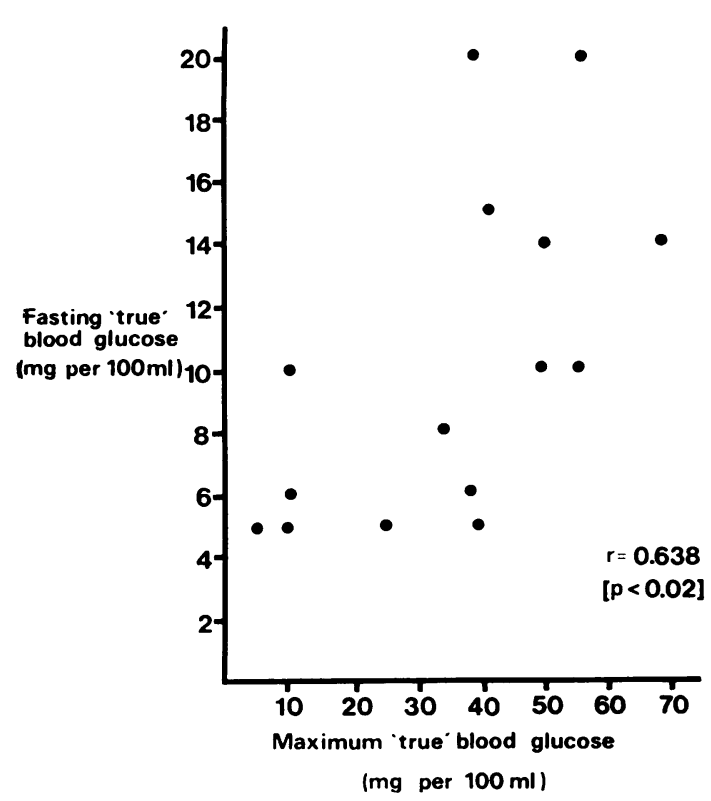

Fig. 1.-The correlation of the initial fasting true blood glucose with the maximum true blood glucose after glucagon stimulation in hypoglycaemic small-for-dates infants.

in which $50 \%$ glucose was used as the stimulus to insulin secretion. The highest maximum insulin recorded was $35 \mu \mathrm{U} / \mathrm{ml}$ in the nonhypoglycaemic group. If this level is taken as the upper limit of normal in the non-hypoglycaemic group, it is possible to divide the hypoglycaemic groups up further into those with a high insulin response and those with a normal insulin response.

Glucagon tests were repeated after recovery at the end of the first week of life in 10 of the infants who had been hypoglycaemic. Tables III and IV compare the glucagon tests in the hypoglycaemic infants before and after treatment. At all times (Table III) the true blood glucose estimations were higher. On the other hand, there was no significant change in the insulin values before and after treatment in the hypoglycaemic group of infants (Table IV).

Tables V to VIII inclusive show the high insulin response group and the normal insulin response group separately, before and after treatment.

Table $\mathrm{V}$ shows that the fasting, 10 minute, 20 minute, and mean maximum true blood glucose estimations were all higher in the normal insulin response group.

Table VII shows the similarity in the high insulin response group: the fasting, 10 minute, 20 minute, 45 minute, 60 minute, and mean maximum true blood glucose estimations were all higher after treatment.

Table VI shows that there was no change in the insulin values before and after treatment in the normal insulin response group. On the other hand, Table VIII shows that in the high insulin response group the fasting, 10 minute, 90 minute, and mean maximum insulin levels were all lower after treatment.

\section{Discussion}

There are only a few reports on the effect of glucagon in hypoglycaemic small-for-dates infants, and some of the results are confusing. Many describe single cases where the response was noted (Cornblath, Odell, and Levin, 1959; Brown and Wallis, 1963; Cornblath et al., 1964; Neligan, 1964). These showed either a negative or weak response to glucagon. Pildes et al. (1967) described a 'satisfactory' response in 4 hypoglycaemic infants, but did not state the initial true blood glucose levels in the individual cases. The correlation

TABLE II

Comparison of Glucagon Tests in Hypoglycaemic and Non-hypoglycaemic Small-for-dates Infants, Insulin $(\mu U / m l)$

\begin{tabular}{|c|c|c|c|c|c|c|c|c|}
\hline \multirow[b]{2}{*}{ Small-for-dates Infants } & \multirow[b]{2}{*}{ No. } & \multicolumn{7}{|c|}{ Time (min) After Injection of Glucagon ( $30 \mu \mathrm{g} / \mathrm{kg}$ ) } \\
\hline & & $\underset{\text { Mean } \pm \text { FD }}{\text { Fange })}$ & $\underset{\substack{\text { Mean } \pm S D \\
(\text { range })}}{10}$ & $\underset{\substack{20 \\
\text { (range) }}}{\operatorname{Mean} \pm S D}$ & $\stackrel{45}{\underset{\text { Mean } \pm S D}{(\text { range })}}$ & $\underset{\substack{\text { Mean } \pm S D \\
(\text { range })}}{60}$ & 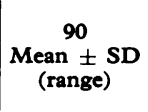 & $\begin{array}{l}\text { Maximum } \\
\text { Insulin } \\
\text { Mean } \pm \text { SD } \\
\text { (range) }\end{array}$ \\
\hline $\begin{array}{l}\text { Hypoglycaemic group } \\
\text { Non-hypoglycaemic group } \\
\text { Significance of difference }\end{array}$ & $\begin{array}{l}14 \\
17 \\
\mathbf{P}\end{array}$ & $\begin{array}{c}20 \cdot 6 \pm 13 \cdot 3 \\
(8-54) \\
12 \cdot 5 \pm 5 \cdot 1 \\
(<5-21) \\
<0 \cdot 5\end{array}$ & $\begin{array}{c}25 \cdot 0 \pm 18 \cdot 9 \\
(8-61) \\
15 \cdot 3 \pm 6 \cdot 9 \\
(5-32) \\
\text { NS }\end{array}$ & $\begin{array}{c}23 \cdot 4 \pm 17 \cdot 3 \\
(9-70) \\
14 \cdot 3 \pm 5 \cdot 3 \\
(<5-22) \\
\text { NS }\end{array}$ & $\begin{array}{l}21 \cdot 6 \pm 14 \cdot 2 \\
(11-58) \\
14 \cdot 5 \pm 5 \cdot 3 \\
(<5-22) \\
\text { NS }\end{array}$ & $\begin{array}{l}20 \cdot 8 \pm 15 \cdot 5 \\
(7-62) \\
13 \cdot 1 \pm 5 \cdot 0 \\
(5-21) \\
\text { NS }\end{array}$ & $\begin{array}{l}20 \cdot 1 \pm 13 \cdot 4 \\
(9-48) \\
13 \cdot 9 \pm 5 \cdot 2 \\
<(5-21) \\
\text { NS }\end{array}$ & $\begin{array}{l}27 \cdot 9 \pm 19 \cdot 0 \\
(11-70) \\
17 \cdot 8 \pm 5 \cdot 6 \\
(10-32) \\
<0 \cdot 05\end{array}$ \\
\hline
\end{tabular}

NS, not significant. 


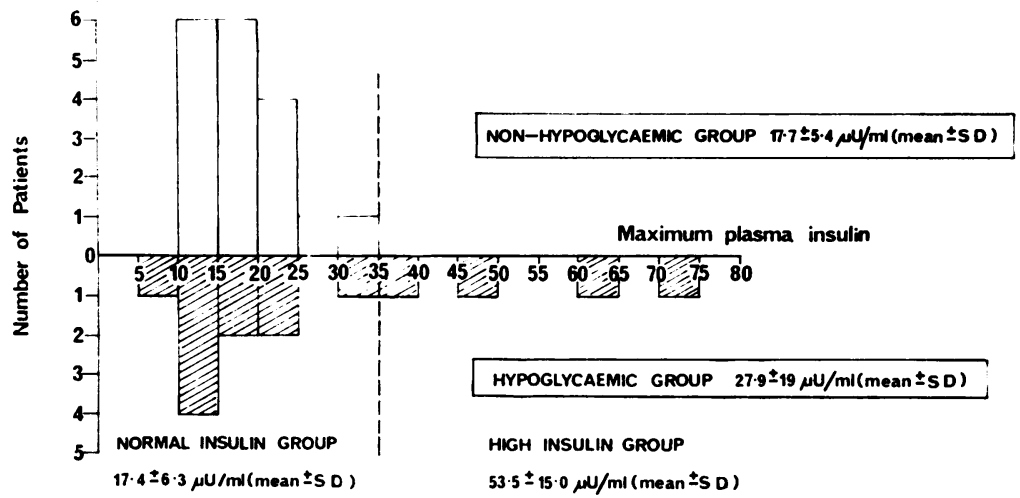

Fig. 2.--Maximum insulin levels after glucagon stimulation in 14 hypoglycaemic and 17 non-hypoglycaemic small-for-dates infants.

\section{TABLE III}

Comparison of Glucagon Tests Before and After Treatment in Hypoglycaemic Small-for-dates Infants, True Blood Glucose $(\mathrm{mg} / 100 \mathrm{ml})$

\begin{tabular}{|c|c|c|c|c|c|c|c|c|}
\hline \multirow[b]{2}{*}{$\begin{array}{c}\text { Small-for-dates } \\
\text { Hypoglycaemic Infants }\end{array}$} & \multirow[b]{2}{*}{ No. } & \multicolumn{7}{|c|}{ Time (min) After Intramuscular Injection of Glucagon $(30 \mu \mathrm{g} / \mathrm{kg})$} \\
\hline & & $\begin{array}{c}\text { Fasting } \\
\text { Mean } \pm \text { SD }\end{array}$ & $\stackrel{10}{\text { Mean } \pm S D}$ & $\stackrel{20}{\text { Mean }} \pm$ SD & $\stackrel{45}{\text { Mean }^{4} \pm \text { SD }}$ & $\stackrel{60}{M I a n} \pm S D$ & $\stackrel{90}{\text { Mean } \pm S D}$ & $\begin{array}{c}\text { Maximum } \\
\text { True Blood } \\
\text { Glucose } \\
\text { Mean } \pm \text { SD }\end{array}$ \\
\hline $\begin{array}{l}\text { Before treatment } \\
\text { After treatment } \\
\text { Significance of difference }\end{array}$ & $\begin{array}{l}15 \\
10\end{array}$ & $\begin{array}{c}10 \cdot 2 \pm 5 \cdot 3 \\
(5-20) \\
37 \cdot 6 \pm 12 \cdot 7 \\
(16-60) \\
<0 \cdot 001\end{array}$ & $\begin{array}{c}17 \cdot 9 \pm 10 \cdot 8 \\
(5-38) \\
52 \cdot 9 \pm 18 \cdot 3 \\
(23-88) \\
<0 \cdot 001\end{array}$ & $\begin{array}{c}24 \cdot 3 \pm 15 \cdot 9 \\
(5-50) \\
62 \cdot 1 \pm 16 \cdot 8 \\
(32-96) \\
<0 \cdot 001\end{array}$ & $\begin{array}{c}34 \cdot 9 \pm 18 \cdot 7 \\
(5-66) \\
61 \cdot 9 \pm 19 \cdot 8 \\
(31-102) \\
<0 \cdot 005\end{array}$ & $\begin{array}{c}30 \cdot 9 \pm 18 \cdot 2 \\
(5-68) \\
50 \cdot 9 \pm 15 \cdot 8 \\
(36-78) \\
<0 \cdot 01\end{array}$ & $\begin{array}{c}29 \cdot 3 \pm 16 \cdot 1 \\
(5-54) \\
42 \cdot 0 \pm 8 \cdot 9 \\
(30-48) \\
<0.05\end{array}$ & $\begin{array}{c}35 \cdot 3 \pm 19 \cdot 7 \\
(5-68) \\
68 \cdot 0 \pm 17 \cdot 7 \\
(32-102) \\
<0 \cdot 001\end{array}$ \\
\hline
\end{tabular}

TABLE IV

Comparison of Glucagon Tests Before and After Treatment in Hypoglycaemic Small-for-dates Infants, Insulin $(\mu U / m l)$

\begin{tabular}{|c|c|c|c|c|c|c|c|c|}
\hline \multirow{2}{*}{$\begin{array}{c}\text { Small-for-dates } \\
\text { Hypoglycaemic Infants }\end{array}$} & \multirow{2}{*}{ No. } & \multicolumn{7}{|c|}{ Time (min) After Intramuscular Injection of Glucagon $(30 \mu \mathbf{g} / \mathbf{k g})$} \\
\hline & & $\begin{array}{c}\text { Fasting } \\
\text { Mean } \pm \text { SD }\end{array}$ & $\stackrel{10}{\text { Mean } \pm S D}$ & $\stackrel{20}{\text { Mean } \pm \text { SD }}$ & $\stackrel{45}{\text { Mean }^{2} \pm S D}$ & $\stackrel{60}{\text { Mean } \pm \text { SD }}$ & Mean $^{90}$ SD & $\begin{array}{c}\text { Maximum } \\
\text { Insulin } \\
\text { Mean } \pm \text { SD }\end{array}$ \\
\hline $\begin{array}{l}\text { Before treatment } \\
\text { After treatment } \\
\text { Significance of difference }\end{array}$ & $\begin{array}{l}14 \\
10 \\
P\end{array}$ & $\begin{array}{c}20 \cdot 6 \pm 13 \cdot 3 \\
(8-54) \\
13 \cdot 4 \pm 4 \cdot 2 \\
(7-22) \\
\text { NS }\end{array}$ & $\begin{array}{c}25 \cdot 0 \pm 18 \cdot 9 \\
(8-61) \\
15 \cdot 8 \pm 7 \cdot 3 \\
(5-31) \\
\text { NS }\end{array}$ & $\begin{array}{c}23 \cdot 4 \pm 17 \cdot 3 \\
(9-70) \\
16 \cdot 2 \pm 7 \cdot 6 \\
(8-32) \\
\text { NS }\end{array}$ & $\begin{array}{c}21 \cdot 6 \pm 14 \cdot 2 \\
(11-58) \\
14 \cdot 8 \pm 6 \cdot 2 \\
(6-26) \\
\text { NS }\end{array}$ & $\begin{array}{c}20 \cdot 8 \pm 15 \cdot 5 \\
(7-62) \\
14 \cdot 1 \pm 6 \cdot 0 \\
(5-24) \\
\text { NS }\end{array}$ & $\begin{array}{c}20 \cdot 1 \quad 13 \cdot 3 \\
(9-48) \\
11 \cdot 1 \pm 3 \cdot 1 \\
(6-15) \\
\text { NS }\end{array}$ & $\begin{array}{c}27 \cdot 9 \pm 18 \cdot 9 \\
(11-70) \\
19 \cdot 5 \pm 7 \cdot 3 \\
(8-32) \\
\text { NS }\end{array}$ \\
\hline
\end{tabular}

NS, not significant.

of the initial fasting true blood glucose with the maximum true blood glucose after glucagon stimulation in the hypoglycaemic infants (Fig. 2) suggests that the degree of hypoglycaemia is dependant upon the severity of the depletion of the glycogen stored in the infant. This could explain the variability of the results obtained in other reports.

In the 28 small-for dates infants described by Blum et al. (1969) only 5 had glucose levels below $20 \mathrm{mg} / 100 \mathrm{ml}$; thus the remaining 23 infants would be in the non-hypoglycaemic group of the 
Comparison of Glucagon Tests in Small-for-dates Hypoglycaemic Infants with Normal Insulin Response Before and After Treatment, True Blood Glucose (mg/100 $\mathrm{ml}$ )

\begin{tabular}{|c|c|c|c|c|c|c|c|c|}
\hline \multirow{2}{*}{$\begin{array}{l}\text { Small-for-dates } \\
\text { Hypoglycaemic } \\
\text { Infants With } \\
\text { Normal Insulin } \\
\text { Response }\end{array}$} & \multirow[b]{2}{*}{ No. } & \multicolumn{6}{|c|}{ Time (min) After Injection of Glucagon $(30 \mu \mathrm{g} / \mathrm{kg})$} & \multirow[b]{2}{*}{$\begin{array}{l}\text { Maximum } \\
\text { True Blood } \\
\text { Glucose } \\
\text { Mean } \pm S D \\
\text { (range) }\end{array}$} \\
\hline & & $\begin{array}{l}\text { Fasting } \\
\text { Mean } \pm \text { SD } \\
\quad \text { (range })\end{array}$ & $\underset{\substack{10 \\
\text { Mean } \pm S D}}{S D}$ & $\underset{\text { Mean } \pm S D}{20}$ & $\underset{\quad \text { Mean } \pm S D}{45}$ & 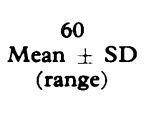 & $\begin{array}{c}90 \\
\text { Mean } \pm S D \\
(\text { range })\end{array}$ & \\
\hline $\begin{array}{l}\text { Before treatment } \\
\text { After treatment } \\
\text { Significance of difference }\end{array}$ & $\begin{array}{r}11 \\
7\end{array}$ & $\begin{array}{l}10 \cdot 9 \pm 5 \cdot 7 \\
(5-20) \\
32 \cdot 9 \pm 11 \cdot 0 \\
(16-50) \\
<0 \cdot 001\end{array}$ & $\begin{array}{l}18 \cdot 4 \pm 9 \cdot 7 \\
(5-32) \\
49 \cdot 7 \pm 16 \cdot 4 \\
(23-70) \\
<0 \cdot 001\end{array}$ & $\begin{array}{l}25 \cdot 3 \pm 14 \cdot 7 \\
\quad(5-46) \\
59 \cdot 1 \pm 14 \cdot 2 \\
(32-70) \\
<0 \cdot 001\end{array}$ & $\begin{array}{l}39 \cdot 2 \pm 17 \cdot 6 \\
(5-66) \\
53 \cdot 4 \pm 13 \cdot 9 \\
(31-78) \\
\text { NS }\end{array}$ & $\begin{array}{l}34 \cdot 4 \pm 18 \cdot 6 \\
(5-68) \\
44 \cdot 0 \pm 11 \cdot 9 \\
(25-60) \\
\text { NS }\end{array}$ & $\begin{array}{c}33 \cdot 2 \pm 15 \cdot 6 \\
(5-54) \\
39 \cdot 7 \pm 9 \cdot 0 \\
(30-53) \\
\text { NS }\end{array}$ & $\begin{array}{l}38 \cdot 8 \pm 19 \cdot 4 \\
\quad(5-68) \\
62 \cdot 1 \pm 15 \cdot 1 \\
(32-78) \\
<0 \cdot 02\end{array}$ \\
\hline
\end{tabular}

NS, not significant.

TABLE VI

Comparison of Glucagon Tests in Small-for-dates Hypoglycaemic Infants with Normal Insulin Response Before and After Treatment, Insulin $(\mu \mathrm{U} / \mathrm{ml})$

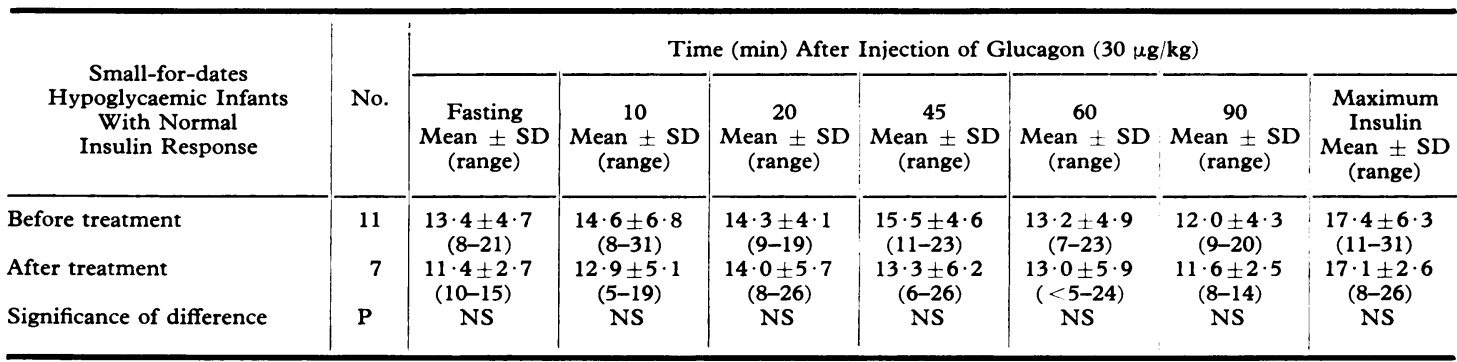

NS, not significant.

TABLE VII

Comparison of Glucagon Tests in Small-for-dates Hypoglycaemic Infants with High Insulin Response Before and After Treatment, True Blood Glucose $(\mathrm{mg} / 100 \mathrm{ml})$

\begin{tabular}{|c|c|c|c|c|c|c|c|c|}
\hline \multirow[b]{2}{*}{$\begin{array}{l}\text { Small-for-dates } \\
\text { Hypoglycaemic } \\
\text { Infants with a } \\
\text { High Insulin } \\
\text { Response }\end{array}$} & \multirow[b]{2}{*}{ No. } & \multicolumn{6}{|c|}{ Time (min) After Injection of Glucagon $(30 \mu \mathrm{g} / \mathrm{kg})$} & \multirow[b]{2}{*}{$\begin{array}{l}\text { Maximum } \\
\text { 'True' Blood } \\
\text { Glucose } \\
\text { Mean } \pm \text { SD } \\
\text { (range) }\end{array}$} \\
\hline & & $\begin{array}{l}\text { Fasting } \\
\text { Mean } \pm \text { SD } \\
\quad(\text { range })\end{array}$ & $\underset{\substack{10 \\
\text { Meange })}}{\stackrel{S D}{\text { range }}}$ & 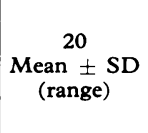 & $\underset{\substack{\text { Mean } \pm S D \\
\text { (range) }}}{45}$ & $\begin{array}{c}60 \\
\underset{(\text { range })}{\text { Mean } \pm S D}\end{array}$ & 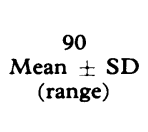 & \\
\hline $\begin{array}{l}\text { Before treatment } \\
\text { After treatment } \\
\text { Significance of difference }\end{array}$ & $\begin{array}{l}4 \\
3\end{array}$ & $\begin{array}{c}8 \cdot 3 \pm 4 \cdot 0 \\
(6-14) \\
48 \cdot 7 \pm 9 \cdot 9 \\
(42-60) \\
<0 \cdot 001\end{array}$ & $\begin{array}{c}18 \cdot 8 \pm 13 \cdot 8 \\
(5-38) \\
60 \cdot 3 \pm 23 \cdot 9 \\
(46-88) \\
<0.05\end{array}$ & $\begin{array}{c}31 \cdot 8 \pm 18 \cdot 5 \\
(5-50) \\
69 \cdot 0 \pm 23 \cdot 8 \\
(51-96) \\
<0.05\end{array}$ & $\begin{array}{c}28 \cdot 3 \pm 15 \cdot 8 \\
(9-46) \\
81 \cdot 7 \pm 18 \cdot 2 \\
(67-102) \\
<0 \cdot 02\end{array}$ & $\begin{array}{l}28 \cdot 3 \pm 13 \cdot 8 \\
(9-40) \\
67 \cdot 0 \pm 11 \cdot 5 \\
(55-78) \\
<0 \cdot 02\end{array}$ & $\begin{array}{c}26 \cdot 0 \pm 14 \cdot 7 \\
(6-38) \\
46 \cdot 7 \pm 8 \cdot 1 \\
(38-54) \\
\text { NS }\end{array}$ & $\begin{array}{c}32 \cdot 8 \pm 17 \cdot 2 \\
(9-50) \\
81 \cdot 7 \pm 18 \cdot 2 \\
(67-102) \\
<0 \cdot 02\end{array}$ \\
\hline
\end{tabular}

NS, not significant.

present series, and therefore would be expected to show a normal response to glucagon. Of the 5 cases with a true blood glucose below $20 \mathrm{mg} / 100 \mathrm{ml}$, 4 were only mildly hypoglycaemic compared with the present series, and therefore would be expected to show a normal or near normal response to gluca- gon. The remaining case gave zero levels or true blood glucose on the second and fifth day of life. Since in the present series all cases of hypoglycaemia were diagnosed within 6 hours of birth, it is difficult to compare this single case.

The fact that the glucagon tests showed a signifi- 
TABLE VIII

Comparison of Glucagon Tests in Small-for-dates Hypoglycaemic Infants with High Insulin Response Before and After Treatment, Insulin $(\mu \mathrm{U} / \mathrm{ml})$

\begin{tabular}{|c|c|c|c|c|c|c|c|c|}
\hline \multirow{2}{*}{$\begin{array}{c}\text { Small-for-dates } \\
\text { Hypoglycaemic Infants } \\
\text { with a High } \\
\text { Insulin Response }\end{array}$} & \multirow[b]{2}{*}{ No. } & \multicolumn{7}{|c|}{ Time (min) After Injection of Glucagon $(30 \mu \mathrm{g} / \mathrm{kg}$ ) } \\
\hline & & $\underset{\substack{\text { Fasting } \\
\text { (range) }}}{\text { Sean }}$ & $\underset{\substack{\text { Mean } \pm S D \\
(\text { range })}}{10}$ & $\underset{\substack{\text { Mean } \pm S D \\
\text { (range) }}}{20}$ & $\underset{\text { (range) }}{45}$ & $\underset{\substack{\text { Mean } \pm S D \\
\text { (range) }}}{60}$ & $\underset{\substack{\text { (range) }}}{\stackrel{90}{\text { Mean }} \pm \mathrm{SD}}$ & $\begin{array}{l}\underset{\text { Maximum }}{\text { Insulin }} \\
\text { Mean } \pm \text { SD } \\
\quad \text { (range) }\end{array}$ \\
\hline $\begin{array}{l}\text { Before treatment } \\
\text { After treatment } \\
\text { Significance of difference }\end{array}$ & 3 & $\begin{array}{l}38 \cdot 0 \pm 11 \cdot 9 \\
(26-54) \\
18 \cdot 0 \pm 3 \cdot 6 \\
(15-22) \\
<0 \cdot 05\end{array}$ & $\begin{array}{l}51 \cdot 0 \pm 11 \cdot 9 \\
(36-61) \\
22 \cdot 7 \pm 7 \cdot 6 \\
(16-21) \\
<0 \cdot 02\end{array}$ & $\begin{array}{l}41 \cdot 8 \pm 19 \cdot 6 \\
(25-70) \\
21 \cdot 3 \pm 10 \cdot 1 \\
(12-32) \\
\text { NS }\end{array}$ & $\begin{array}{l}38 \cdot 0 \pm 19 \cdot 5 \\
(19-58) \\
18 \cdot 3 \pm 5 \cdot 7 \\
(12-23) \\
\text { NS }\end{array}$ & $\begin{array}{l}37 \cdot 8 \pm 18 \cdot 5 \\
(19-62) \\
16 \cdot 7 \pm 6 \cdot 4 \\
(12-24) \\
\text { NS }\end{array}$ & $\begin{array}{l}32 \cdot 2 \pm 13 \cdot 2 \\
(19-48) \\
10 \cdot 3 \pm 4 \cdot 5 \\
(6-15) \\
<0 \cdot 05\end{array}$ & $\begin{array}{l}53 \cdot 5 \pm 15 \cdot 0 \\
(36-70) \\
25 \cdot 0 \pm 6 \cdot 1 \\
(21-32) \\
<0.05\end{array}$ \\
\hline
\end{tabular}

NS, not significant.

cant change at the end of the first week of life, and that the hypoglycaemia had disappeared concurrently in these infants, support the view that these two factors must be related.

Though there is a clear division between the high and normal insulin response groups in this paper, there does not appear to be any correlation between the degree of glycogen depletion and the type of insulin response. The response to glucagon is variable in neonatal hypoglycaemia, and it therefore cannot be recommended as a therapeutic tool in clinical practice in this situation.

I thank Professor J. H. Hutchison and Dr. I. D. Riley for help in the preparation of this paper, and Dr. R. W. Logan and Miss O. Peden of the Biochemistry Department, Royal Hospital for Sick Children, Glasgow, for much assistance.

\section{REFERENCES}

Blum, D., Dodion, J., Loeb, H., Wilkin, P., and Hubinont, P. O. (1969). Studies on hypoglycaemia in small-for-dates newborns. Archives of Disease in Childhood, 44, 304.

Brown, R. J. K., and Wallis, P. G. (1963). Hypoglycaemia in the newborn infant. Lancet, 1, 1278.

Cornblath, M., Odell, G. B., and Levin, E. Y. (1959). Symptomatic neonatal hypoglycaemia associated with toxaemia of pregancy. Fournal of Pediatrics, 55, 545.

Cornblath, M., and Schwartz, R. (1966a). Carbohydrate homeostasis in the neonate (full term and low birthweight). In Disorders of Carbohydrate Metabolism in Infancy, p. 33. Saunders, Philadelphia and London.
Cornblath, M., and Schwartz, R. (1966b). Disorders of Carbohydrate Metabolism in Infancy, p. 82. Saunders, Philadelphia and London.

Cornblath, M., Wybregt, S. H., Baens, G. S., and Klein, R. I. (1964). Symptomatic neonatal hypoglycaemia. VIII. Studies of carbohydrate metabolism in the newborn infant. Pediatrics, $33,388$.

Dawkins, M. J. R. (1964). Hypoglycaemia in childhood. Proceedings of the Royal Society of Medicine, 57, 1063.

Hales, C. N., and Randle, P. J. (1963). Immunoassay of insulin with insulin-antibody precipitate. Biochemical fournal, 88, 137.

Le Dune, M. A. (1971). Intravenous glucose tolerance and plasma insulin studies in small-for-dates infants. Archives of Disease in Childhood, 47, 111.

Marks, V. (1959). An improved glucose-oxidase method for determining blood, C.S.F. and urine glucose levels. Clinica Chemica Acta, 4, 395.

Milner, R. D. G., and Wright, A. D. (1967). Plasma glucose, nonesterified fatty acid, insulin and growth hormone response to glucagon in the newborn. Clinical Science, 32, 249.

Neligan, G. A. (1964). Hypoglycaemia in the newborn infant. In Nutricia Symposium on The Adaption of the Newborn Infant to Extra-Uterine Life, p. 44. Ed. by J. H. P. Jonxis, H. K. A. Visser, and J. A. Troelestra. Stenfert Kroese, N.V., Leiden.

Pildes, R., Forbes, A. E., O'Connor, S. M., and Cornblath, M (1967). The incidence of neonatal hypoglycaemia: a completed survey. Fournal of Pediatrics, 70, 76.

Shelley, H. J. (1964). Carbohydrate reserves in the newborn infant. British Medical fournal, 1, 273.

Shelley, H. J., and Neligan, G. A. (1966). Neonatal hypoglycaemia. British Medical Bulletin, 22, 34.

Tanner, J. M., and Thomson, A. M. (1970). Standards for birthweight at gestation periods from 32 to $\mathbf{4 2}$ weeks, allowing for maternal height and weight. Archives of Disease in Childhood, 45, 566.

Correspondence to Dr. M. A. Le Dune, Department of Child Health, Royal Hospital for Sick Children, Yorkhill, Glasgow G3 8SJ. 